\title{
Nanoscale
}

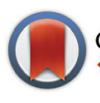

CrossMark $\leftarrow$ click for updates

Cite this: Nanoscale, 2016, 8, 4529

Received 3rd July 2015, Accepted 5th December 2015

DOI: $10.1039 / c 5 n r 04439 a$

www.rsc.org/nanoscale

\section{New insights into colloidal gold flakes: structural investigation, micro-ellipsometry and thinning procedure towards ultrathin monocrystalline layers $\uparrow$}

\author{
B. Hoffmann, ${ }^{\ddagger}{ }^{a}$ M. Y. Bashouti, ${ }^{a}{ }^{a}$ T. Feichtner, ${ }^{\text {ba }}$ a. Mačković, ${ }^{c}$ C. Dieker, ${ }^{c}$ \\ A. M. Salaheldin, ${ }^{d}$ P. Richter, ${ }^{e}$ O. D. Gordan, ${ }^{e}$ D. R. T. Zahn, ${ }^{e}$ E. Spiecker ${ }^{\mathrm{C}}$ and \\ S. Christiansen ${ }^{\text {b.a }}$
}

\begin{abstract}
High-quality fabrication of plasmonic devices often relies on wet-chemically grown ultraflat, presumably single-crystalline gold flakes due to their superior materials properties. However, important details about their intrinsic structure and their optical properties are not well understood yet. In this study, we present a synthesis routine for large flakes with diameters of up to $70 \mu \mathrm{m}$ and an in-depth investigation of their structural and optical properties. The flakes are precisely analyzed by transmission electron microscopy, electron backscatter diffraction and micro-ellipsometry. We found new evidence for the existence of twins extending parallel to the Au flake $\{111\}$ surfaces which have been found to not interfere with the presented nanopatterning. Micro-Ellipsometry was carried out to determine the complex dielectric function and to compare it to previous measurements of bulk single crystalline gold. Finally, we used focused ion beam milling to prepare smooth crystalline layers and high-quality nanostructures with desired thickness down to $10 \mathrm{~nm}$ to demonstrate the outstanding properties of the flakes. Our findings support the plasmonics and nano optics community with a better understanding of this material which is ideally suited for superior plasmonic nanostructures.
\end{abstract}

\section{Introduction}

Plasmonics, the physics of coupled states between light and surface charge density oscillations, ${ }^{1}$ is a thriving research topic with a plethora of potential applications, like optical antennas, ${ }^{2,3}$ plasmonic waveguides, ${ }^{4}$ SPASER $^{5}$ or meta-materials. ${ }^{6}$ For their realization, metallic nanostructures with design and fabrication accuracy at the nanometer scale are needed to

\footnotetext{
${ }^{a}$ Max Planck Institute for the Science of Light, D-91058 Erlangen, Germany. E-mail: bjoern.hoffmann@mpl.mpg.de, muhammad.bashouti@mpl.mpg.de ${ }^{b}$ Helmholtz Zentrum Berlin für Materialien und Energie GmbH, D-14109 Berlin, Germany

${ }^{c}$ Friedrich-Alexander-Universität Erlangen-Nürnberg (FAU), Lehrstuhl für Mikro- und Nanostrukturforschung (WW9) \& Center for Nanoanalysis and Electron Microscopy, Department Werkstoffwissenschaften, D-91058 Erlangen, Germany

${ }^{d}$ Friedrich-Alexander-Universität Erlangen-Nürnberg (FAU), Institute of Particle Technology, D-91058 Erlangen, Germany

${ }^{e}$ Semiconductor Physics, Technische Universität Chemnitz, D-09107 Chemnitz, Germany

$\dagger$ Electronic supplementary information (ESI) available. See DOI: 10.1039/ c5nr04439a

$\$$ B.H. and M.Y.B. contributed equally.
}

ensure functionality at a desired wavelength, while minimizing losses.

Plasmonic structures are often made from noble metals such as gold, as it is stable in ambient conditions and it shows surface plasmon resonances in the visible wavelength range. There are two established routes to prepare gold layers for subsequent nanostructuring: (i) chemical synthesis yielding colloidal metal nanoparticles, ${ }^{7-10}$ which thereafter can be manipulated and arranged to desired layouts; (ii) deposition of gold layers by sputtering, thermal or electron beam evaporation. The structuring can be performed by sophisticated methods like electron beam lithography followed by dry or wet-chemical etching, direct laser lithography ${ }^{11}$ or straightforward direct focused ion beam (FIB) milling. ${ }^{12}$

Deposited gold layers, however, pose difficulties for the fabrication of high-quality plasmonic nanostructures with nanoscale precision due to their intrinsic structure: they are typically nano-crystalline with grain sizes in the range of 10 to several $100 \mathrm{~nm}$ and show related to the granularity a notable surface roughness. Both grain boundaries and roughness affect patterning processes negatively. Grains with varied crystal orientations exhibit different etching rates under 
focused ion beam (FIB) treatment, grain boundaries and other crystal defects lead to an increased FIB etching rate, and a rough surface further influences the local etching rate. ${ }^{13}$ Thus, deposited gold layers show a highly anisotropic etching behaviour which heavily influences the geometrical fabrication precision. ${ }^{8}$ Furthermore, defects and grain boundaries in metallic nanostructures can hamper plasmonic properties due to increased ohmic losses.

Therefore, the ideal substrate for patterning large area, complex shaped plasmonic structures is a thin single-crystalline layer with an atomically smooth surface. While deposited layers cannot ensure the required single crystallinity, large particles with these properties were first reported in 2004, fabricated by wet-chemical synthesis routines. ${ }^{14-16}$ In the following years the synthesis was refined towards reliable mass production $^{17}$ and larger flake sizes. ${ }^{18}$ In 2005 Lofton \& Sigmund proposed a growth mechanism for gold flakes and suggested the existence of single in-plane twins inside the flakes. ${ }^{19}$ Only in 2010 it was realized that these particles are an ideal building block for plasmonic nanostructures when Huang et al. reported on the superiority of atomically flat gold flakes over deposited gold layers in terms of fabrication accuracy and optical quality of prepared plasmonic antennas. ${ }^{8}$ The work of Huang et al. constituted an initial spark for highquality plasmonics and since then many groups have used gold flakes for their plasmonic applications. ${ }^{20-29}$ For example, Dai et al. demonstrated single-crystalline optical nanocircuits with local mode conversion, ${ }^{21}$ Prangsma et al. fabricated electrically connected optical antennas with promising electrooptical properties, ${ }^{25}$ and Kern et al. continued that work and recently presented antennas with electrically tunable scattering spectra. ${ }^{27} \mathrm{Wu}$ et al. have presented a versatile way how to transfer nanostructures that were etched in gold flakes to arbitrary substrates. ${ }^{28}$ Feichtner $e t$ al. have shown the fabrication of complex plasmonic nanoantennas that follow an evolutionary design. ${ }^{29}$ All those papers would not have been possible in that quality with vapor-deposited gold.

However, only little effort was carried out to investigate the nanostructure as well as the optical properties of wet-chemically synthesized gold flakes in detail. Furthermore, several articles have indicated the existence of planar defects inside the flakes, ${ }^{19,30}$ but to the best of our knowledge no direct evidence was provided until now. Here, we present a detailed transmission electron microscopy study that reveals yet unknown details about the presence of multiple twin boundaries inside the $\mathrm{Au}$ flakes. Furthermore, we carried out micro-ellipsometry on individual flakes to determine their complex dielectric function and show that it is comparable to previous measurements on polished mono-crystalline bulk material. ${ }^{25}$ Finally, we gradually thinned and nanostructured a flake to prepare nanostructures as thin as $10-15 \mathrm{~nm}$ which are still single-crystalline as shown by electron backscatter diffraction (EBSD). The successful FIB thinning procedure provides now for the option to realize gold nano-patterns of presumably any thickness. Our work is intended to shed light on the remaining secrets of ultraflat gold flakes and to promote their advantages and utilization to a broader community.

\section{Experimental}

\section{Chemical synthesis}

Our wet-chemical synthesis of colloidal gold flakes is based on an already established recipe ${ }^{7}$ with customized solution concentrations and process temperature. $0.82 \mathrm{mmol}(0.28 \mathrm{~g})$ of hydrogen tetrachloroaurate trihydrate $\left(\mathrm{HAuCl}_{4} \cdot 3 \mathrm{H}_{2} \mathrm{O}\right)$ are added to $50 \mathrm{ml}$ of ethylene glycol and stirred for $20 \mathrm{~min}$ in a single-necked flask $(100 \mathrm{ml})$ at $100{ }^{\circ} \mathrm{C}$. The slurry is reduced by adding $0.14 \mathrm{mmol}$ of Aniline $(1.4 \mathrm{ml})$ at $100{ }^{\circ} \mathrm{C}$ and the stirring is stopped after 5 minutes. Then the mixture is kept in a temperature-controlled electro-mantle for 180 minutes. Afterwards, the flask is mildly sonicated to remove particles that adhered to the flask walls. The suspension is then centrifuged and re-suspended in ethanol several times to clean and purify the gold particles. Our synthesis produces triangular and hexagonal flakes with sizes of up to $70 \mu \mathrm{m}$ and thicknesses between $30-80 \mathrm{~nm}$. In contrast to the recipe that we loosely follow, ${ }^{7}$ our modified parameters lead to a large increase in average particle size.

If the suspension is stored for a long time ( $>$ three months), the flakes will grow on and their thickness will increase substantially; for some of these long-term grown Au flakes thickness values of 400 to $1000 \mathrm{~nm}$ were observed. This is ideal for ellipsometry, as the data evaluation can be carried out assuming bulk gold within the model. Since visible light has a penetration depth into gold of up to $80 \mathrm{~nm}$, thin flakes would require a modelling that takes the underlying material into consideration thereby making the fitting procedure more complex.

\section{Transmission electron microscopy}

Transmission electron microscopy (TEM) was carried out with an FEI Titan ${ }^{3}$ Themis 300 and a Philips CM30 TWIN/STEM. The $\operatorname{Titan}^{3}$ Themis is equipped with a high-brightness fieldemission gun (X-FEG), a monochromator system, two $\mathrm{C}_{\mathrm{S}}$-correctors (probe and image side) from CEOS, a Super-X detector for energy dispersive X-ray (EDX) spectroscopy, a high-angle annular dark-field (HAADF) detector and a 4k CMOS camera. The CM30 TWIN/STEM is equipped with a $\mathrm{LaB}_{6}$ filament. Both TEMs were operated at $300 \mathrm{kV}$ acceleration voltage. Convergent beam electron diffraction (CBED) was used to determine the thickness of the Au flakes, while selected area electron diffraction (SAED), dark field (DF) TEM and scanning TEM (STEM) imaging was used for a detailed structural analysis. In general, for TEM analysis the Au flakes are prepared by drop-casting on copper TEM grids coated with a continuous carbon film. TEM images and diffraction patterns are acquired by using a charge coupled device camera from TVIPS (Tietz Video and Image Processing Systems GmbH, Germany) and a 4k CMOS camera.

The evaluation of the diffraction patterns and dynamical diffraction simulations were carried out by using the software 
JEMS (version 3.7624U2012) ${ }^{32}$ and the crystal data from the inorganic crystal structure database (ICSD). For a more detailed defect analysis and thickness evaluation of the Au flakes crosssections were prepared by applying a special FIB technique in shadow geometry. ${ }^{33}$ Image analysis was performed by using the free available software ImageJ (version 1.46r) and the commercially available software DigitalMicrograph ${ }^{\mathrm{TM}}$.

\section{Scanning electron microscopy (SEM) and electron backscatter diffraction (EBSD)}

SEM analysis of flake morphology and EBSD investigation of crystal orientation and lattice defects were performed inside a dual beam FIB/SEM (TESCAN Lyra 3) equipped with a Bruker e-Flash ${ }^{\mathrm{HR}}$ EBSD detector. For EBSD, the sample is tilted $70^{\circ}$ and the area of interest is scanned by the electron beam. The backscattered primary electrons, which are partly diffracted by the crystal lattice, are collected by a phosphorous screen which is imaged by a high resolution camera that collects the characteristic Kikuchi patterns. The EBSD software analyses these patterns to determine a $3 \mathrm{D}$ orientation information of each pixel. In this way information on grain structure, grain boundary population, lattice strain and tilt can be derived. Since the silicon substrate is as much crystalline as the gold flake, showing a cubic lattice, the EBSD software is not able to distinguish between $\mathrm{Si}$ and Au. The scanning parameters were thus chosen in a way that the EBSD camera recognizes the Kikuchi pattern from $\mathrm{Au}$, but is not able to resolve the Si patterns $(10 \mathrm{kV}$ acceleration voltage, $1 \mathrm{nA}$ beam current, $20 \mathrm{~ms}$ per pixel mapping speed).

\section{Focused ion beam milling}

FIB milling was carried out in the same microscope as described in the section before. A FIB acceleration voltage of $30 \mathrm{kV}$ and an ion beam current of $25 \mathrm{pA}$ were used. The patterning was done with a pixel dwell time of $1 \mu$ s and a step size of $5 \mathrm{~nm}$.

\section{Ellipsometry}

The ellipsometric measurements were done using an Accurion "nanofilm ep4" (Accurion GmbH, Göttingen, Germany) imaging ellipsometer operating with the nulling principle ${ }^{34}$ in a PCA (polarizer, compensator, [sample], analyzer) configuration (see ESI $\dagger$ for details). In nulling ellipsometry, the ellipsometric parameters $\Psi$ and $\Delta$ are calculated from the PCA rotational angles for which the light intensity at the detector is zero. The spectra were acquired under a $50 \times$ microscope objective in a spectral region from 1.55 to $3.25 \mathrm{eV}$ with a $0.02 \mathrm{eV}$ energy step at a $40^{\circ}$ and $50^{\circ}$ angle of incidence. $\Psi$ and $\Delta$ were extracted only from a small region of interest positioned in the middle of the gold flake.

The obtained experimental data was evaluated using the WVASE32 $2^{\mathrm{TM}}$ software. ${ }^{35}$ The sample was approximated as a half infinite isotropic medium, covered by a $2.5 \mathrm{~nm}$ thick transparent layer represented by a Cauchy dispersion function, taking account for organics and water physisorbed on the top of the flake. An optical model consisting of a Drude contribution and
3 Gaussian oscillators was applied, to assure the KramersKronig consistency of the spectra and to further compare the data to other literature reports. By fitting this parametric model to the experimental values, the resulting dielectric function $\varepsilon=\varepsilon^{\prime}+i \varepsilon^{\prime \prime}$ for the investigated single crystalline Au flake could be extracted.

\section{Results \& discussion}

\section{Structural investigation}

TEM was used to characterize the nanostructure of the $\mathrm{Au}$ flakes. Fig. 1(a) and (c) exemplarily show two as-synthesized Au flakes. The contrast variations which are visible in these bright-field (BF) TEM images are related to bend contours. The corresponding (inverted) SAED patterns are shown in Fig. 1(b) and (d), respectively.

Both SAED patterns are valid for the [111] zone axis (ZA) of the face-centered cubic (fcc) Au and also consistent with ICSD 44362. From the SAED pattern in Fig. 1(b) it is evident that the Au flake in Fig. 1(a) is single-crystalline. In contrast, the SAED pattern in Fig. 1(d) additionally exhibits weak 1/3 $\{422\}$ type reflections next to the regular strong reflections. These reflections can be attributed to several reasons such as monoatomic surface steps, surface reconstructions or stacking faults/twins. In 1993, Kirkland et al. investigated the reasons for the occurrence of formally forbidden reflections in small Au flakes in a)
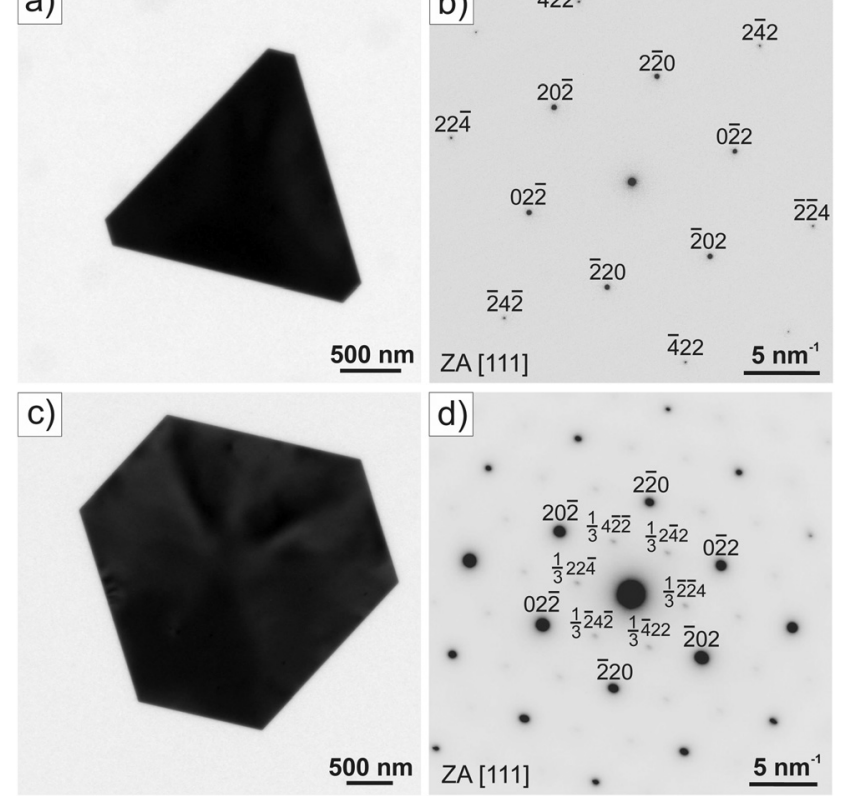

Fig. 1 TEM analysis of as-synthesized Au flakes. In (a) and (c) exemplary $\mathrm{BF}$ TEM images of two Au flakes are shown; (b) and (d) represent (inverted) SAED patterns of the Au flakes shown in (a) and (c), respectively. Both SAED patterns are valid for the [111] ZA of Au (consistent with ICSD 44362). The weaker $1 / 3\{422\}$ type reflections indicate the presence of planar defects in the Au flakes, which is also shown in FIB crosssections in Fig. 2. 
detail and concluded that the most probable reason is the existence of twins which extend parallel to the flake $\{111\}$ surfaces. ${ }^{36}$ Similar observations were reported by Germain et al. for twinned silver particles. ${ }^{37}$ The authors gave a detailed summary of possible reasons for the occurrence of $1 / 3\{422\}$ type reflections in fcc metals. Hence, these formally forbidden weak reflections are likely due to the presence of twins. To sufficiently prove the existence of the extended lattice defects in more detail, cross-sections of Au flakes were prepared by using a special shadow-FIB technique. ${ }^{33}$ The results are summarized in Fig. 2. In a first step the Au flakes are drop-casted on a piece of silicon wafer and $\mathrm{Au}$ flakes which were found to be stacked on each other are selected for the shadow-FIB preparation. From BF TEM imaging of these cross-sections, as exemplarily shown in Fig. 2(b) and (c) it is evident that the Au flakes exhibit various thicknesses and are either with or without planar defects (dark line contrast clearly visible in the interior of one of the particles). These planar defects are found to extend parallel to the $\{111\}$ surfaces of the Au flakes. Furthermore, some of the Au flakes exhibit point-/island-like contrasts, possibly caused by FIB damage. It is well known that high-energy particle-induced damage can for instance lead to sample surface amorphization, point defect creation and dislocation formation. ${ }^{13,38,39}$ However, in the present work it can be excluded that the planar defects arise from FIB preparation, since we observed the presence of these planar defects by electron diffraction and HRTEM even before FIB preparation (see Fig. 1 and $\mathrm{ESI}_{\dagger} \dagger$ ).

Fig. 2(d) shows two typical Au flakes in [110] viewing direction. The corresponding SAED pattern in [110] ZA is shown in Fig. 2(e) and confirms the presence of twin boundaries of first order (equals to so called $\sum=3$ twins according to the coinci-



Fig. 2 Defect and thickness analysis of the Au flakes: (a) SEM image of Au flakes prepared on a piece of silicon wafer for the shadow-FIB preparation of TEM cross-sections; the BF TEM images in (b) and (c) exemplarily show TEM cross-sections of the Au flakes located in the region which is marked with the red dotted rectangular in (a); (d) is a BF TEM image of two Au flakes in [110] viewing direction, while the corresponding SAED pattern in [110] ZA is shown in (e). DF TEM images in ( $f$ ) and ( $g$ ) are obtained for the same Au flakes shown in (d); (h) is a BF TEM image with the corresponding experimental CBED pattern in (i). In (j) the simulated CBED pattern of (i) is shown. In (k) the thickness is plotted for twelve Au flakes, obtained by CBED analysis, while (l) plots the thickness of ten different Au flakes, evaluated from TEM cross-sections after preparation by the shadow-FIB technique. 
dent site lattice notation), which as such are characteristic for fcc metals. ${ }^{40}$ In the case of a $\Sigma=3$ twin boundary in fcc metals many pairs of $g$-vectors exactly overlap but have very different indices. ${ }^{40}$ By exemplarily selecting the $(1-1-1)$ and $(002)_{\text {twin }}$ reflections from the SAED pattern and performing DF TEM imaging, both twin counterparts of the Au flakes become evident, as shown in the corresponding DF TEM images in Fig. 2(f) and (g), respectively.

The thickness of the flakes can be determined either by destructive cross-section analysis or by non-destructive CBED experiments and simulations. We have determined the thickness of the Au flakes by using CBED and correlated these results with thickness measurements, which we obtained from TEM imaging of FIB cross-sections. These results are illustrated in Fig. 2(h-l). The contrast variations visible in Fig. 2(h) can be attributed to bend contours. The CBED pattern of the triangular Au flake shown in Fig. 2(h) is depicted in Fig. 2(i) and was obtained in three-beam conditions. In order to evaluate the thickness from such CBED patterns we have used the software JEMS, ${ }^{32}$ whereby the simulated CBED pattern is adapted until matching the experimental CBED pattern.

This is exemplarily shown in Fig. 2(i) and (j), where in this particular case a thickness of $47 \mathrm{~nm}$ is observed for the $\mathrm{Au}$ flake. Fig. 2(k) plots the thickness of altogether twelve Au flakes, which was determined by CBED. As can be seen, the thickness is ranging from $40-47 \mathrm{~nm}$ for the given synthesis procedure. It has to be mentioned that the determination of the thickness by means of CBED is in general limited to a small sample region (depending on the spot size of the electron beam which is used). In our case the electron beam was converged and positioned in the middle parts of the $\mathrm{Au}$ flakes. Consequently, the thickness values obtained from CBED are not representative for the whole Au particle. Therefore, we have evaluated the thickness of ten different Au flake cross sections prepared by shadow FIB and analyzed by TEM, where the measurements are conducted over the whole length of the flakes. These results are shown in Fig. 2(l). From the cross section analysis Au flakes are found to be even thinner, which was not observed in CBED. The error bars in Fig. 2(1) are a measure of the $\mathrm{Au}$ flakes inhomogeneous thickness, which varies over the whole length of the Au flakes. The acquired thickness values from these two different methods don't match perfectly due to a too small dataset. However, our intention is rather to show two different reliable methods of thickness evaluation.

Even though we have proven that the Au flakes can contain twins that exist throughout the whole particles, their effect on both patterning and plasmonic properties is expected to be negligible for the vast majority of possible structures. $\Sigma=$ 3 twin boundaries are represented by an inversion in stacking order of the atomic planes from ABCABCABC to ABC|BACBA. Such a change in stacking order will most likely not interfere with FIB etching. Furthermore, the planar defects are found to be always parallel to the $\{111\}$ surfaces of the Au flakes and perpendicular to the incident ions, which will result in a homogeneous behavior during nanopatterning. The plasmonic properties will also not be negatively affected by the twins because in most applications the electrons oscillate parallel to the twin planes, and thus they don't 'see' the defects and possible ohmic losses on them.

The existence of twins is already determined by the structure of the seed particle. If it has twins, they will continue into the flake which grows out of it. It is still not well understood what type of seed particles promote the existence of twins. Kirkland et al. suggested a bi-tetrahedron with 3 -fold symmetry. ${ }^{36}$ Lofton \& Sigmund proposed a different growth mechanism for gold flakes in which triangular particles morelikely contain one twin while hexagonal flakes contain two or more twins tending to reach much larger sizes. ${ }^{19}$ However, we cannot confirm this model because the largest particles we typically find are triangular. Furthermore, we have not found any relation between the flake shape and the amount of twins.

In addition to the SAED and DF-TEM investigations shown in Fig. 1 and 2 we have performed high-resolution TEM of as-prepared flakes as well as high-resolution scanning TEM (HRSTEM) analysis of the flake cross sections. Both analyses are presented in the ESI $\dagger$ and further support our here presented findings.

A fast and large scale investigation of the crystallinity of the flakes has been performed by means of EBSD, which is a surface sensitive diffraction method carried out in a SEM. Fig. 3(a) shows an SEM image of three overlapping flakes which were deposited by drop casting on a silicon substrate. The Inverse Pole Figure (IPF) map from the EBSD analysis in Fig. 3(b) shows the crystal facet pointing in $x$-direction (IPF- $x$ ). The flakes have different orientations due to the random
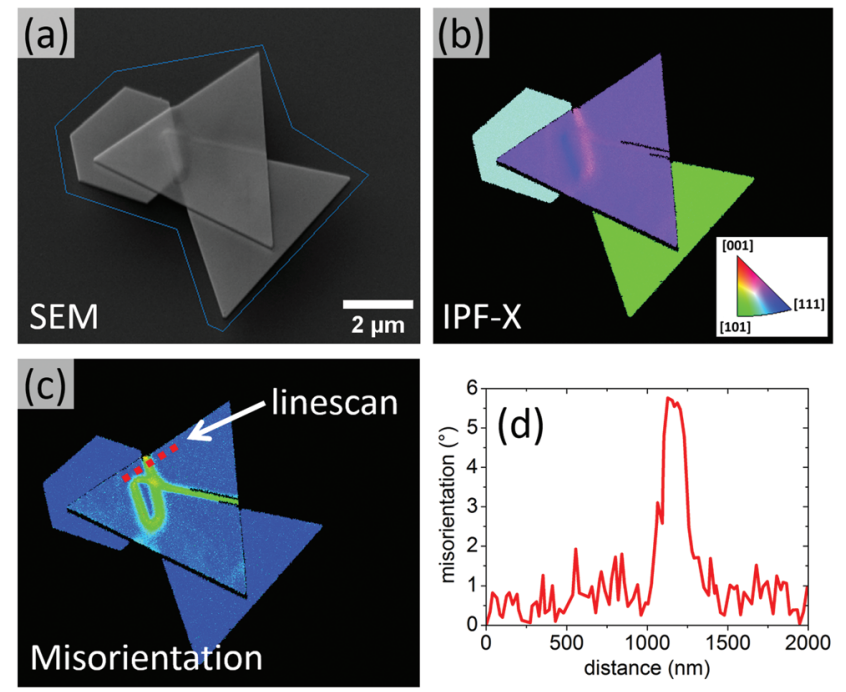

Fig. 3 SEM images of three overlapping Au flakes (a) and the corresponding maps that are calculated from the EBSD measurement. The IPF- $x$ map (b) proves that the flakes are single crystalline. The misorientation map (c) shows the stress inside the top flake due to local bending on the flakes underneath. The red line in (c) corresponds to the misorientation graph in (d). Although the top flake is tilted more than $5^{\circ}$ in a length of only $100 \mathrm{~nm}$, it still remains single crystalline. 
deposition mechanism, but apart from that are perfectly monocrystalline in all areas. Bending due to the overlap induced stress is well visible in Fig. 3(c), the flakes' misorientation map. A misorientation linescan, shown in Fig. 3(d), shows that the flakes can bend as much as $5-6^{\circ}$ in a very short distance without breaking and still remain monocrystalline. Combining TEM results and surface sensitive, large scale EBSD measurements (see $\mathrm{ESI}_{\dagger} \dagger$ for additional information) we can state that the $\mathrm{Au}$ flakes exhibit a perfect crystalline quality, occasional twins will most likely not deteriorate the nanopatterning.

\section{Micro-ellipsometry}

In addition to the structural information about the flakes, the optical properties are also highly important for plasmonic applications. However, the determination of dielectric constants of such small particles is not trivial. For the first time, it was possible to perform micro-ellipsometry (see Methods) within areas as small as $5 \mu^{2}$ on a $400 \mathrm{~nm}$ thick gold flake with lateral dimensions of about $15 \mu \mathrm{m}$ shown in Fig. 4(a) and (b). The small roughness which is visible in the AFM linescan in Fig. 4(b) can be attributed to physisorbed organics which are treated by the Cauchy layer during fitting. The fit works best when an atomically flat gold layer is assumed which supports the assumption of an ultraflat gold surface.

The large thickness of the flake originates from a long storage time of three months without cleaning the stock solution. This allows the treatment of the material as half infinite isotropic medium simplifying data interpretation. The retrieved optical constants were fitted with a Drude model contribution and three Gaussian oscillators (see Methods). Fig. 4(c) shows the resulting dielectric constants (solid curves) compared to the experimental values for polished monocrystalline gold from Olmon et al. ${ }^{31}$ (dashed curves). Both real and imaginary part are comparable to the established experimental values. There is no observable effect of the lower surface roughness of the flakes, compared to the monocrystalline data of Olmon et al., where polishing left roughness in the range of $1.12 \mathrm{~nm}$ as well as larger geometrical features from polishing. The $\mathrm{Au}$ flakes show a roughness below $0.5 \mathrm{~nm}$ as grown (compare with Fig. 2c) and FIB milled $^{8}$ and their surface is not indented by any polishing means allowing controlled structuring on its full surface.

Chemically grown gold flakes surpass evaporated as well as template stripped poly crystalline gold (materials used also by Olmon et $a l^{31}$ ), as they combine the best of both materials: essentially no surface roughness, which avoids undesired coupling channels between plasmons and far-field radiation as well as no inter-grain boundaries, which give rise to unintentional plasmon to phonon coupling.

\section{Thinning \& patterning}

Local thinning of an $\mathrm{Au}$ layer can be of valuable interest for 3-dimensional patterning or ultrathin compact layers, since a $10 \mathrm{~nm}$ thin deposited layer is not yet continuous. To test the influence of thinning on the properties of the layer and a)

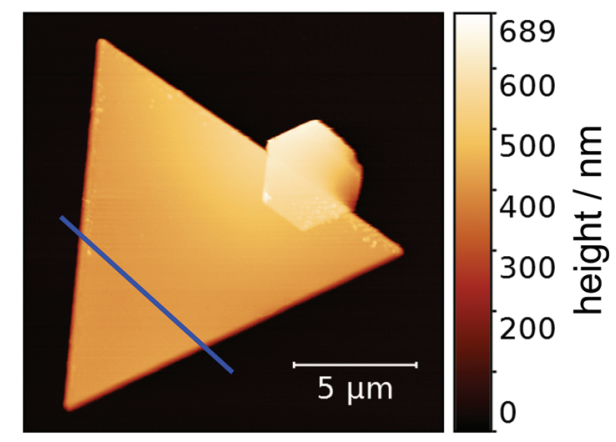

b)

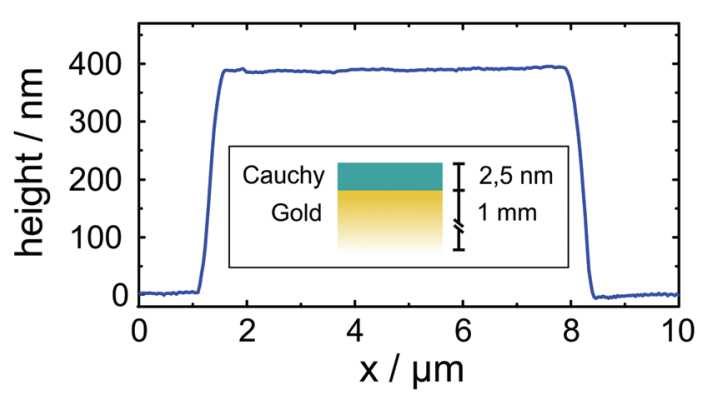

C)

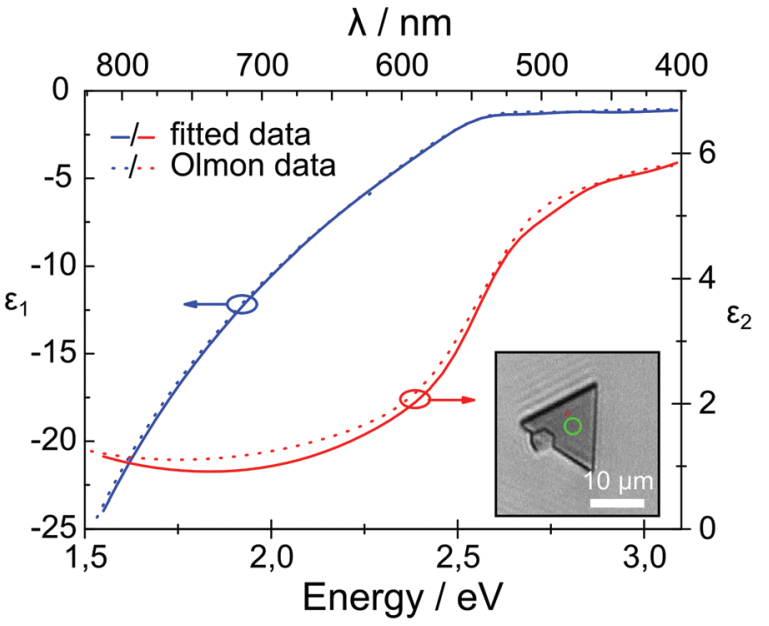

Fig. 4 Ellipsometry on gold flakes: (a) AFM image of the flake. (b) Height profile along the blue line in (a). The inset shows the layer model which is used for ellipsometry. (c) Complex dielectric function (blue: real part; red: imaginary part) of monocrystalline gold flakes (solid line) compared with values of recent data for monocrystalline gold from Olmon et al. ${ }^{31}$ (dashed line). The measurement area is shown in the inset optical microscope image.

subsequent nanostructure formation, we used a $60 \mathrm{~nm}$ thin, large $\mathrm{Au}$ flake and a $60 \mathrm{~nm}$ thin layer of sputtered $\mathrm{Au}$ as comparison. Rectangles with sizes of $3 \times 3 \mu \mathrm{m}^{2}$ and an etching depth of 0 to $55 \mathrm{~nm}$ in steps of $5 \mathrm{~nm}$ were etched into both layer types.

Fig. 5(a) and (c) show a comparison between the sputtered layer and the Au flake by coloured SEM images, directly after etching. It is clearly visible that the etching of the polycrystalline film yields a very rough Au layer. In contrast, the thinning of a monocrystalline flake produces very smooth surfaces. After the thinning process a second etching step was 
Sputtered Au layer
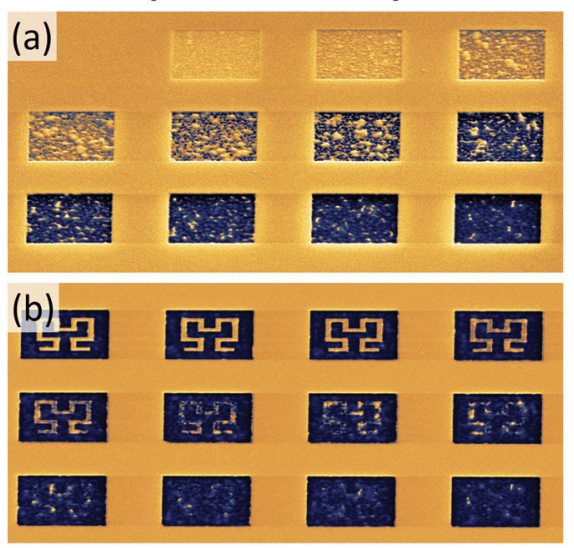

Au flake
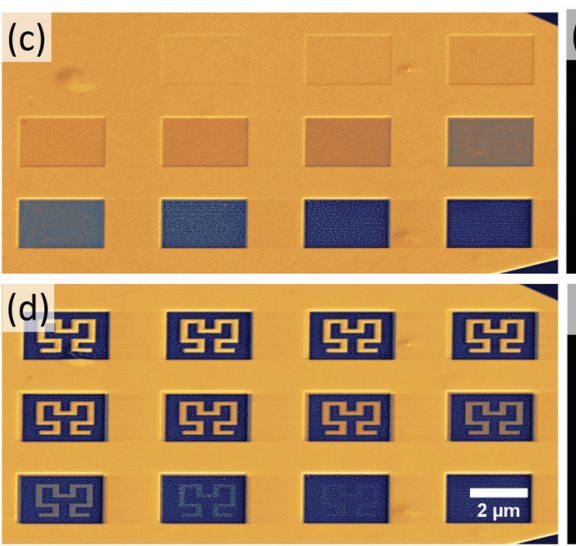

EBSD on (d)

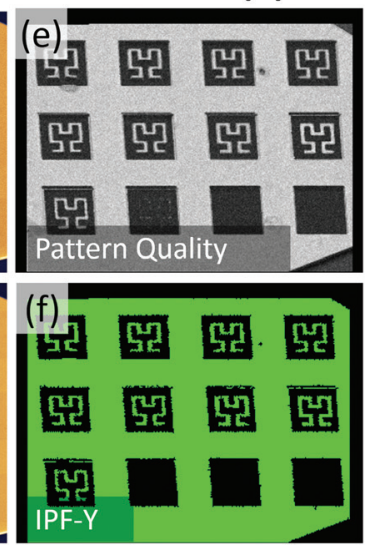

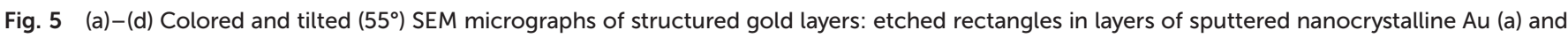

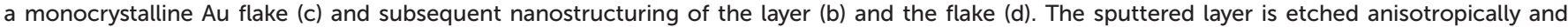

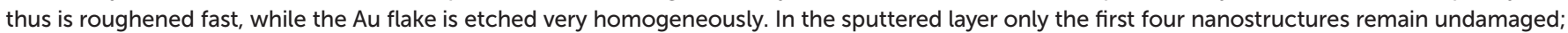



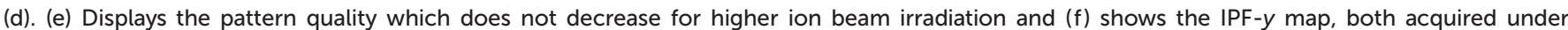
$70^{\circ}$ angle.

performed to realize nanosized Hilbert curves with feature sizes of $200 \mathrm{~nm}$ at controlled thicknesses. Fig. 5(b) and (d) show that the antenna structures in a nanocrystalline Au layer have good quality only for larger layer thicknesses. A thinning of $20 \mathrm{~nm}$ ( $5^{\text {th }}$ field) is already leading to a damaged structure with irregular line profiles. On the other hand, antenna structures in thinned Au flakes remain excellent in shape down to a thickness of 10-15 $\mathrm{nm}$.

We performed EBSD scans to evaluate the influence of the FIB etching on the crystalline properties. Fig. 5(e) and (f) collect the result of EBSD on the thinned and nanostructured $\mathrm{Au}$ flake. The thinning does not destroy the crystallinity and even the last intact structure in the bottom left with a thickness of 10-15 $\mathrm{nm}$ remains monocrystalline, even though the high energy gallium ions have an interaction range in $\mathrm{Au}$ of about $10 \mathrm{~nm}$. The large amount of energy deposited in the material due to the impact and recoil of $\mathrm{Ga}^{+}$-ions is incapable of destroying the crystallinity of the Au flakes. Thus, we are able to prepare ultrathin complex nanostructures with an unmatched crystalline quality.

Being able to homogeneously etch gold over large areas, 3-dimensional nanostructuring with high accuracy becomes possible. For example, plasmonic antennas that make use of plasmon focusing not only in $x$ - and $y$-direction, but also in $z$-direction could lead to higher efficient sensors. The ESI (Fig. S3†) contains two examples of accurate 3D nanopatterning.

\section{Conclusions}

We have presented an in-depth analysis of yet unknown structural and optical properties of widely used gold flakes, which constitute an ideal building block for plasmonic applications. We have shown the presence of twins in a fraction of flakes which were only predicted, but never investigated in detail until now. The twins are expected to have negligible negative influence on the plasmonic properties or on the precise nanopatterning.

The flakes optical properties are at least comparable to polished single crystalline bulk gold unhampered by intrinsic grain boundaries with the additional benefit of minimal surface roughness. The ideal optical properties, together with the superior nanopatterning behaviour, explains why this material is of such great importance for high-quality plasmonic applications.

The most important finding is that even a $10 \mathrm{~nm}$ thin FIB-polished $\mathrm{Au}$ flake still remains crystalline and can be structured in a subsequent etching step to generate ultrathin plasmonic devices. Using a combination of bottom-up and top-down nanofabrication, it is possible to obtain complex plasmonic nanostructures with well-defined dimensions and crystallographic orientation, as well as atomically flat surfaces. It can be applied to all kinds of substrates and may thus facilitate hybrid plasmonic-waveguiding (using $\mathrm{Si}$ as substrate for example) and plasmonic lasing, in which a well-controlled deep sub-wavelength contact between different materials is necessary.

\section{Acknowledgements}

The authors gratefully acknowledge financial support by the European Union Seventh Framework Programme (FP7/20072013) under the Grant Agreement no 280566, project UnivSEM, by the DFG Research Training Group GRK1896 "InSitu Microscopy with Electrons, X-rays and Scanning Probes" and by the DFG Cluster of Excellence EXC315 "Engineering of Advanced Materials (EAM)”. M.Y.B. gratefully acknowledges 
the Max-Planck Society for the Post-Doctoral fellowship. J. Müller is acknowledged for additional microscopy support.

\section{Notes and references}

1 S. A. Maier, Plasmonics: fundamentals and applications, Springer, 2007.

2 P. Biagioni, J. S. Huang and B. Hecht, Rep. Prog. Phys., 2012, 75, 024402.

3 B. Hoffmann, S. Vassant, X.-W. Chen, S. Götzinger, V. Sandoghdar and S. Christiansen, Nanotechnology, 2015, 26, 404001.

4 D. K. Gramotnev and S. I. Bozhevolnyi, Nat. Photonics, 2010, 4, 83-91.

5 D. J. Bergman and M. I. Stockman, Phys. Rev. Lett., 2003, 90, 027402.

6 C. M. Soukoulis and M. Wegener, Science, 2010, 330, 16331634.

7 Z. Guo, Y. Zhang, Y. DuanMu, L. Xu, S. Xie and N. Gu, Colloids Surf., A, 2006, 278, 33-38.

8 J.-S. Huang, V. Callegari, P. Geisler, C. Brüning, J. Kern, J. C. Prangsma, X. Wu, T. Feichtner, J. Ziegler, P. Weinmann, M. Kamp, A. Forchel, P. Biagioni, U. Sennhauser and B. Hecht, Nat. Commun., 2010, 1, 150.

9 S. Gwo, M.-H. Lin, C.-L. He, H.-Y. Chen and T. Teranishi, Langmuir, 2012, 28, 8902-8908.

10 Y. Jin, Adv. Mater., 2012, 24, 5153-5165.

11 S. Bagheri, K. Weber, T. Gissibl, T. Weiss, F. Neubrech and H. Giessen, ACS Photonics, 2015, 2, 779-786.

12 T. Coenen, F. Bernal Arango, A. Femius Koenderink and A. Polman, Nat. Commun., 2014, 5, 3250.

13 C. A. Volkert and A. M. Minor, MRS Bull., 2007, 32, 389-399.

14 X. Sun, S. Dong and E. Wang, Angew. Chem., Int. Ed., 2004, 43, 6360-6363.

15 J.-U. Kim, S.-H. Cha, K. Shin, J. Y. Jho and J.-C. Lee, Adv. Mater., 2004, 16, 459-464.

16 Y. Shao, Y. Jin and S. Dong, Chem. Commun., 2004, 1104-1105.

17 C. Li, W. Cai, B. Cao, F. Sun, Y. Li, C. Kan and L. Zhang, Adv. Funct. Mater., 2006, 16, 83-90.

18 X. Sun, S. Dong and E. Wang, Langmuir, 2005, 21, 47104712.

19 C. Lofton and W. Sigmund, Adv. Funct. Mater., 2005, 15, 1197-1208.
20 C. Y. Wu, C. T. Kuo, C. Y. Wang, C. L. He, M. H. Lin, H. Ahn and S. Gwo, Nano Lett., 2011, 11, 4256-4260.

21 W. H. Dai, F. C. Lin, C. Bin Huang and J. S. Huang, Nano Lett., 2014, 14, 3881-3886.

22 W. Chen, F. Lin, Y. Lee, F. Li, Y. Chang and J.-S. Huang, ACS Nano, 2014, 8, 9053-9062.

23 P. Geisler, G. Razinskas, E. Krauss, X.-F. Wu, C. Rewitz, P. Tuchscherer, S. Goetz, C.-B. Huang, T. Brixner and B. Hecht, Phys. Rev. Lett., 2013, 111, 183901.

24 F. Pashaee, F. Sharifi, G. Fanchini and F. LagugnéLabarthet, Phys. Chem. Chem. Phys., 2015, 17, 21315-21322.

25 J. C. Prangsma, J. Kern, A. G. Knapp, S. Grossmann, M. Emmerling, M. Kamp and B. Hecht, Nano Lett., 2012, 12, 3915-3919.

26 S. Viarbitskaya, A. Teulle, R. Marty, J. Sharma, C. Girard, A. Arbouet and E. Dujardin, Nat. Mater., 2013, 12, 426-432.

27 J. Kern, R. Kullock, J. Prangsma, M. Emmerling, M. Kamp and B. Hecht, Nat. Photonics, 2015, 9, 582-586.

28 X. Wu, P. Geisler, E. Krauss, R. Kullock and B. Hecht, Nanoscale, 2015, 16427-16433.

29 T. Feichtner, O. Selig and B. Hecht, 2015, arXiv:1511.05438 [physics.optics].

30 J. J. Diao and H. Chen, J. Chem. Phys., 2006, 124, 116103.

31 R. L. Olmon, B. Slovick, T. W. Johnson, D. Shelton, S.-H. Oh, G. D. Boreman and M. B. Raschke, Phys. Rev. B: Condens. Matter, 2012, 86, 235147.

32 P. Stadelmann, Java version 3.8431U2012, CIME-EPFL, Jems Electron Microscopy Software, Switzerland, 1999-2012.

33 B. F. Vieweg, B. Butz, W. Peukert, R. N. Klupp Taylor and E. Spiecker, Ultramicroscopy, 2012, 113, 165-170.

34 R. M. Azzam and N. M. Bashara, Ellipsometry and Polarized Light, North-Holland Pub. Co., 1977.

35 B. Johs, C. Herzinger and B. Guenther, WVASE32 Ver. 3.668, J.A. Woollam Co., Lincoln, NE, USA.

36 A. I. Kirkland, D. A. Jefferson, D. G. Duff, P. P. Edwards, I. Gameson, B. F. G. Johnson and D. J. Smith, Proc. R. Soc. London, Ser. A, 1993, 440, 589-609.

37 V. Germain, J. Li, D. Ingert, Z. L. Wang and M. P. Pileni, J. Phys. Chem. B, 2003, 107, 8717-8720.

38 T. H. Blewitt, R. R. Coltman and J. K. Redman, J. Appl. Phys., 1957, 28, 651-660.

39 J. A. Venables, Philos. Mag., 1961, 6, 379-396.

40 D. B. Williams and C. B. Carter, Transmission Electron Microscopy - A Textbook for Materials Science, Springer, 2nd edn, 2009. 